PROCEEDINGS OF THE

AMERICAN MATHEMATICAL SOCIETY

Volume 126, Number 1, January 1998, Pages 195-201

S 0002-9939(98)03483-2

\title{
WEAKLY CONVERGENT SEQUENCE COEFFICIENT IN KÖTHE SEQUENCE SPACES
}

\author{
YUNAN CUI
}

(Communicated by Palle E. T. Jorgensen)

\begin{abstract}
In this paper, we have discussed the weakly convergent sequence coefficient in Köthe sequence spaces with $\left(e_{n}\right)$ as their boundedly complete basis. Using those results, we can easily calculate the weakly convergent sequence coefficient in Orlicz sequence spaces.
\end{abstract}

\section{INTRODUCTION}

Our aim is to calculate the weakly convergent sequence coefficient in the Köthe sequence spaces. The weakly convergent sequence coefficient concerned with normal structure is an important geometric parameter (see [1], [2], [10], [8]). It was introduced by Bynum (see [1]). In the sequel, $X$ denotes a Banach space and $S(X)$ denotes the unit sphere of $X . l^{0}$ stands for the space of all infinite real sequences. Let $N$ and $R$ be the set of natural numbers and the set of real numbers, respectively.

Definition 1. The weakly convergent sequence coefficient of $X$, denoted $\operatorname{WCS}(X)$, is defined as follows:

$\operatorname{WCS}(X)=\sup \left\{k:\right.$ for each weakly convergent sequence $\left\{x_{n}\right\}_{n=1}^{\infty}$,

there exists some $y \in \operatorname{co}\left(\left\{x_{n}\right\}\right)$ such that $\left.k \cdot \limsup _{n \rightarrow \infty}\left\|x_{n}-y\right\| \leq A\left(\left\{x_{n}\right\}\right)\right\}$;

here $\operatorname{co}\left(\left\{x_{n}\right\}\right)$ denotes the convex hull of the elements of $\left\{x_{n}\right\}_{n=1}^{\infty}$.

It is easy to see that $1 \leq \operatorname{WCS}(X) \leq 2$ (see [1]). The notion of normal structure has been introduced by Brodskic and Millman in [4] connected with the fixed point theory. For a bounded subset $A$ of $X$, the Chebyshev self-radius of $A$ is the number

$$
r(A)=\inf \{\sup \{\|x-y\|: y \in A\}: x \in \operatorname{co}(A)\} .
$$

Definition 2. A Banach space $X$ is said to have normal structure if $r(A)<$ $\operatorname{Diam}(A)$ for every non-singleton bounded subset $A$ of $X$.

It is known that a Banach space with normal structure has the fixed point property (see [1], [3], [4], [9]), and every reflexive Banach space with $\operatorname{WCS}(X)>1$

Received by the editors September 19, 1994 and, in revised form, May 19, 1995, April 22, 1996, and July 15, 1996.

1991 Mathematics Subject Classification. Primary 46B20, 46B30, 46E20.

Key words and phrases. Weakly convergent sequence coefficient, Köthe sequence spaces.

The author was supported by the NSF and ECF of China.

(C)1998 American Mathematical Society 
has normal structure (see [1]). For a sequence $\left\{x_{n}\right\}_{n=1}^{\infty}$ of $X$, we define

$$
A\left(\left\{x_{n}\right\}\right)=\limsup _{n \rightarrow \infty}\left\{\left\|x_{i}-x_{j}\right\|: i, j \geq n, i \neq j\right\}
$$

and

$$
A_{1}\left(\left\{x_{n}\right\}\right)=\liminf _{n \rightarrow \infty}\left\{\left\|x_{i}-x_{j}\right\|: i, j \geq n, i \neq j\right\} .
$$

Definition 3. A sequence $\left\{x_{n}\right\}$ is said to be an asymptotic equidistant sequence if $A\left(\left\{x_{n}\right\}\right)=A_{1}\left(\left\{x_{n}\right\}\right)($ see $[2])$.

The result that $\operatorname{WCS}(X)=\inf \left\{A\left(\left\{x_{n}\right\}\right):\left\{x_{n}\right\}\right.$ a sequence in $S(X)$ and $x_{n} \stackrel{w}{\longrightarrow}$ $0\}=\inf \left\{A\left(\left\{x_{n}\right\}\right):\left\{x_{n}\right\}\right.$ an asymptotic equidistant sequence in $S(X)$ and $\left.x_{n} \stackrel{w}{\longrightarrow} 0\right\}$ is obtained in [2].

Definition 4. A Banach space $X$ is said to have weakly uniformly normal structure if $\operatorname{WCS}(X)>1$ (see [7]).

A complete normed sequence space $X$ is called a Köthe sequence space if $x=$ $(x(1), x(2), \ldots) \in l^{0}$ and $y=(y(1), y(2), \ldots) \in X$ such that $|x(i)| \leq|y(i)|$ for all $i \in N$, then $x \in X$ and $\|x\| \leq\|y\|$.

Definition 5. A map $M: R \rightarrow R$ is called an Orlicz function if it satisfies the following conditions:

(1) $M$ is even, continuous, convex, and $M(0)=0$ if and only if $u=0$;

(2) $\lim _{u \rightarrow \infty} \frac{M(u)}{u}=\infty$ and $\lim _{u \rightarrow 0} \frac{M(u)}{u}=0$.

For every Orlicz function $M$ we define the complementary function $M^{*}: R \rightarrow R$ by the formula $M^{*}(\nu)=\sup _{u>0}\{u|\nu|-M(u)\}$ for every $\nu \in R$.

An Orlicz sequence space $l_{M}$ generated by $M$ is defined as follows:

$$
l_{M}=\left\{x \in l^{0}: R_{M}(k x)=\sum M(k x(i))<\infty \text { for some } k>0\right\} .
$$

The Orlicz sequence spaces $l_{M}$ are considered as Banach spaces equipped with the Luxemburg norm $\|x\|_{(M)}=\inf \left\{k>0: R_{M}\left(\frac{x}{k}\right) \leq 1\right\}$, or with the Orlicz-Amemiya norm $\|x\|_{M}=\inf \left\{\frac{1}{k}\left(1+R_{M}(k x)\right): k>0\right\}$.

To simplify notation, we put $l_{(M)}=\left(l_{M},\|\|_{(M)}\right)$ and $l_{M}=\left(l_{M},\|\|_{M}\right)$.

Definition 6. We say an Orlicz function $M$ satisfies the $\delta_{2}$-condition $\left(M \in \delta_{2}\right.$, for short) if there exist constants $K \geq 2$ and $u_{0}>0$ such that

$$
M(2 u) \leq K M(u) \quad \text { for every }|u| \leq u_{0} .
$$

For more details about Orlicz spaces, we refer the reader to [5], [9], [11] or [12].

\section{REsults}

Theorem 1. Let $X$ be a Köthe sequence space with $\left\{e_{n}\right\}$ as its boundedly complete basis, where $e_{n}=(0,0, \ldots, 0, \stackrel{n t h}{1}, 0, \ldots)$. Then

$$
\mathrm{WCS}(X)=\inf \left\{A\left(\left\{x_{n}\right\}\right): x_{n}=\sum_{i=I_{(n-1)}+1}^{I_{n}} x_{n}(i) e_{i}, x_{n} \stackrel{w}{\longrightarrow} 0, I_{1}<I_{2}<\cdots\right\} .
$$


Proof. Let $d=\inf \left\{A\left(\left\{x_{n}\right\}\right): x_{n}=\sum_{i=I_{(n-1)}+1}^{I_{n}} x_{n}(i) e_{i}, x_{n} \stackrel{w}{\longrightarrow} 0, I_{1}<I_{2}<\cdots\right\}$. We need only show that $\operatorname{WCS}(X) \geq d$.

For any $\varepsilon>0$, by the definition of $\operatorname{WCS}(X)$, there exists an asymptotic equidistant sequence $\left\{x_{n}\right\}$ in $S(X)$ with $x_{n} \stackrel{w}{\longrightarrow} 0$ such that $A\left(\left\{x_{n}\right\}\right)<\operatorname{WCS}(X)+\varepsilon$.

By $x_{n} \stackrel{w}{\longrightarrow} 0$, we have that

$$
x_{n}(i) \rightarrow 0 \quad \text { as } n \rightarrow \infty(i=1,2, \ldots) .
$$

Let $v_{1}=x_{1}$ and take $I_{1} \in N$ so that $\left\|\sum_{i=I_{1}}^{\infty} v_{1}(i) e_{i}\right\|<\varepsilon$. By (1) we can choose $N_{1} \in N$ such that

$$
\left\|\sum_{i=1}^{I_{1}} x_{n}(i) e_{i}\right\|<\varepsilon
$$

whenever $n \geq N_{1}$.

Let $v_{2}=x_{N_{1}}$ and take $I_{2}>I_{1}$ such that $\left\|\sum_{i=I_{2}+1}^{\infty} v_{2}(i) e_{i}\right\|<\varepsilon$. By (1), we can find $N_{2}>N_{1}$ so that for $n \geq N_{2}$,

$$
\left\|\sum_{i=1}^{I_{2}} x_{n}(i) e_{i}\right\|<\varepsilon
$$

Let $v_{3}=x_{N_{2}}$ and take $I_{3}>I_{2}$, so that $\left\|\sum_{i=I_{3}+1}^{\infty} v_{3}(i) e_{i}\right\|<\varepsilon$.

In such a way, we can obtain a sequence $\left\{I_{n}\right\}$ of positive integers with $0<I_{1}<$ $I_{2}<\ldots$, and a subsequence $\left\{v_{n}\right\}$ of $\left\{x_{n}\right\}$ satisfying $A\left(\left\{x_{n}\right\}\right)=A\left(\left\{v_{n}\right\}\right)$ and

$$
\left\|\sum_{i=1}^{I_{n-1}} v_{n}(i) e_{i}\right\|<\varepsilon, \quad\left\|\sum_{i=I_{n}+1}^{\infty} v_{n}(i) e_{i}\right\|<\varepsilon, \quad n=1,2,3, \ldots,
$$

where $I_{0}=0$.

Hence, for any $n<m$ we have

$$
\begin{aligned}
\| v_{n}- & v_{m} \| \\
= & \left\|\left(\sum_{i=1}^{I_{(n-1)}}+\sum_{i=I_{(n-1)}+1}^{I_{n}}+\sum_{i=I_{n}+1}^{I_{(m-1)}}+\sum_{i=I_{(m-1)}+1}^{I_{m}}+\sum_{i=I_{m}+1}^{\infty}\right)\left(v_{n}(i)-v_{m}(i)\right) e_{i}\right\| \\
\geq & \left\|\left(\sum_{i=I_{(n-1)}+1}^{I_{n}}+\sum_{i=I_{(m-1)}+1}^{I_{m}}\right)\left(v_{n}(i)-v_{m}(i)\right) e_{i}\right\| \\
& -\left\|\left(\sum_{i=1}^{\left.I_{(} n-1\right)}+\sum_{i=I_{n}+1}^{I_{(m-1)}}+\sum_{i=I_{m}+1}^{\infty}\right)\left(v_{n}(i)-v_{m}(i)\right) e_{i}\right\| \\
\geq & \left\|\sum_{i=I_{(n-1)}+1}^{I_{n}} v_{n}(i) e_{i}+\sum_{i=I_{(m-1)}+1}^{I_{m}} v_{m}(i) e_{i}\right\|-2 \varepsilon-6 \varepsilon \\
\geq & \left\|\sum_{i=I_{(n-1)}+1}^{I_{n}} u_{n}(i) e_{i}+\sum_{i=I_{(m-1)}+1}^{I_{m}} y_{m}(i) e_{i}\right\|-8 \varepsilon
\end{aligned}
$$

where $u_{n}=\sum_{i=I_{n-1}+1}^{I_{n}} v_{n}(i) e_{i} /\left\|\sum_{i=I_{(n-1)}+1}^{I_{n}} v_{n}(i) e_{i}\right\|$, so $\left\|u_{n}\right\|=1$. 
Thus $A\left(\left\{u_{n}\right\}\right) \leq A\left(\left\{v_{n}\right\}\right)+8 \varepsilon \leq A\left(\left\{x_{n}\right\}\right)+8 \varepsilon \leq \operatorname{WCS}(X)+9 \varepsilon$. In view of the arbitrariness of $\varepsilon>0$, we have $d \leq \operatorname{WCS}(X)$.

Theorem 2. Orlicz sequence spaces $l_{(M)}\left(\right.$ or $\left.l_{M}\right)$ has the weak uniform normal structure if and only if $M \in \Delta_{2}$.

Proof. Necessity. In the case of $X=l_{M}$, for any $0<\varepsilon<1 / 2$, by $\operatorname{WCS}(X)=1$ there exists an asymptotic equidistant sequence $\left\{x_{n}\right\}$ in $S(X)$ with $x_{n} \stackrel{w}{\longrightarrow} 0$ such that $1 \leq A\left(x_{n}\right) \leq 1+\varepsilon$.

Without loss of generality, we may assume $1-\varepsilon \leq\left\|x_{n}-x_{1}\right\|_{M} \leq 1+\varepsilon$.

By $\left\|x_{n}\right\|_{M} \geq 1 / 2$ and $M \in \Delta_{2}$, there exists $\delta>0$ such that

$$
\sum_{i=1}^{\infty} M\left(x_{n}(i)\right) \geq \delta
$$

Hence, when $n>I_{1}$, we get

$$
\begin{aligned}
1+\varepsilon & \geq\left\|x_{n}-x_{1}\right\|_{M} \\
& =\left(1+\sum_{i=1}^{\infty} M\left(k_{n}\left(x_{n}(i)-x_{1}(i)\right)\right)\right) / k_{n} \\
& =\left(1+\sum_{i=1}^{\infty} M\left(k_{n}\left(x_{1}(i)\right)\right)\right) / k_{n}+\sum_{i=1}^{\infty} M\left(k_{n}\left(x_{n}\right)\right) / k_{n} \\
& \geq\left(1+\sum_{i=1}^{\infty} M\left(k_{n}\left(x_{1}(i)\right)\right) / k_{n}\right)+\delta \\
& \geq\left\|x_{1}\right\|_{M}+\delta \geq 1+\delta .
\end{aligned}
$$

This contradiction shows $M \notin \Delta_{2}$.

Sufficiency. By $M \notin \Delta_{2}$, for any $\varepsilon>0$ there is a $u>0$ such that $u<\varepsilon$ and $\varepsilon M((1+\varepsilon) u)>M(u)$. Setting $v=(1+\varepsilon) u$, we get $M(v /(1+\varepsilon))<\varepsilon M(v)$.

Since $v<2 \varepsilon$, we can find a positive integer $m$ such that

$$
1-M(2 \varepsilon)<m M(v) \leq 1 .
$$

Take $c \geq 0$ satisfying $m M(v)+M(c)=1$. Then $M(c)<M(2 \varepsilon)$. Put

$$
\begin{aligned}
& x_{1}=(c, \overbrace{v, \ldots, v}^{m}, 0,0, \ldots), \\
& x_{2}=(\overbrace{0, \ldots, 0}^{m}, c, \overbrace{v, \ldots, v}^{m}, 0,0, \ldots),
\end{aligned}
$$

and $y_{n}=x_{n} /\left\|x_{n}\right\|_{M}$. Then $y_{n} \in S\left(l_{M}\right)$ and $\left\{y_{n}\right\}$ is an asymptotic equidistant sequence.

Next, we will show that $y_{n} \stackrel{w}{\longrightarrow} 0$. It is obvious that $y_{n}(i) \rightarrow 0(i=1,2, \ldots)$. Since

$$
\limsup _{k \rightarrow 0} R_{M}\left(k x_{n}\right) / k=\limsup _{k \rightarrow 0} R_{M}\left(k x_{1}\right) / k=0,
$$

we have $x_{n} \stackrel{l_{M^{*}}}{\longrightarrow} 0$ which means the convergence with respect to regular functionals. It is obvious that $\Phi\left(x_{n}\right)=0$ for any singular functional. Hence, $y_{n} \stackrel{w}{\longrightarrow} 0$. 
Finally, we estimate $A\left(\left\{y_{n}\right\}\right)$. We have

$$
\begin{aligned}
\left\|\left(y_{n}-y_{k}\right) /(1+\varepsilon)\right\|_{M} & =\left\|\left(x_{n}-x_{k}\right) /(1+\varepsilon)\right\| x_{1}\left\|_{M}\right\|_{M} \\
& \leq\left\|\left(x_{n}-x_{k}\right) /(1+\varepsilon)\right\|_{M} \\
& \leq 1+R_{M}\left(\left(x_{n}-x_{k}\right) /(1+\varepsilon)\right) \\
& =1+2 m M(v /(1+\varepsilon))+2 M(c /(1+\varepsilon)) \\
& \leq 1+2 m \varepsilon M(v)+2 M(c) \\
& <1+2 \varepsilon+2 M(2 \varepsilon) .
\end{aligned}
$$

This means that $A\left(\left\{y_{n}\right\}\right)<(1+\varepsilon)(1+2 \varepsilon+2 M(2 \varepsilon))$, i.e., $\operatorname{WCS}\left(l_{M}\right)=1$.

The proof of the case $X=l_{(M)}$ is similar to that of $X=l_{M}$.

Recalling that Maluta's coefficient $D(X)$ of a Banach space $X$ is defined by [10] $D(X)=\sup \left\{\lim \sup d\left(x_{n+1},\left\{x_{i}\right\}_{i=1}^{n}\right) / A\left(\left\{x_{n}\right\}\right): x_{n}\right.$ a bounded nonconstant sequence in $X\}$ and $\operatorname{WCS}(X)=1 / D(X)$ for reflexive spaces $X$ (cf. [13]) and $D(X)=1$ for nonreflexive spaces $X$ (cf. [10]), we obtain that there exists a Banach space $X$ such that $\operatorname{WCS}(X) \neq D(X)$.

Theorem 3. If $M$ satisfies the $\Delta_{2}$-condition, we have

$$
\operatorname{WCS}\left(l_{(M)}\right)=\inf \left\{c>0: \sum_{i=1}^{n} M\left(u_{1} / c\right)=1 / 2, \sum_{i=1}^{n} M\left(u_{i}\right)=1, n=1,2, \ldots\right\} .
$$

Proof. Let $d=\inf \left\{c>0: \sum_{i=1}^{n} M\left(u_{i} / c\right)=1 / 2, \sum_{i=1}^{n} M\left(u_{i}\right)=1, n=1,2, \ldots\right\}$. For any $\left(u_{1}, u_{2}, \ldots, u_{n}\right)$ satisfying $\sum_{i=1}^{n} M\left(u_{i}\right)=1$, define

$$
x_{m}=(\overbrace{0, \ldots, 0}^{m n}, u_{1}, u_{2}, \ldots, u_{n}, 0, \ldots) \quad(m=1,2, \ldots) .
$$

Obviously, $x_{m} \stackrel{w}{\longrightarrow} 0$ and $\left\|\left(x_{n}-x_{k}\right) / c\right\|_{(M)}=1$ if $n \neq k$, i.e., $A\left(\left\{x_{n}\right\}\right)=c$.

By $M(u) \in \Delta_{2},\left\{e_{n}\right\}$ is a boundedly complete base of $l_{(M)}$, whence $\operatorname{WCS}\left(l_{(M)}\right)$ $\leq d$.

On the other hand, by $M \in \Delta_{2}$, we have $\|x\|_{(M)}=1$ if and only if $R_{M}\left(x_{n}\right)=1$. For any

$$
x_{n}=\sum_{i=I_{(n-1)}+1}^{I_{(n)}} x_{n}(i) e_{i} \in S(X), \quad \text { where } I_{1}<I_{2}<\cdots,
$$

we have $x_{n} \stackrel{w}{\longrightarrow} 0$ and $R_{M}\left(\left(x_{n}-x_{k}\right) / d\right)=R_{M}\left(x_{n} / d\right)+R_{M}\left(x_{k} / d\right) \geq 1(n \neq k)$. This means that $A\left(\left\{x_{n}\right\}\right) \geq d$, i.e., $\operatorname{WCS}\left(l_{(M)}\right) \geq d$.

So, $\operatorname{WCS}\left(l_{(M)}\right)=\inf \left\{c>0: \sum_{i=1}^{n} M\left(u_{i} / c\right)=1 / 2, \sum_{i=1}^{n} M\left(u_{i}\right)=1, n=\right.$ $1,2, \ldots\}$.

Corollary 1. For $l_{p}(1<p<\infty)$ we have $\operatorname{WCS}\left(l_{p}\right)=2^{1 / p}$ (see [1] and [10]).

Theorem 4. If $M \in \Delta_{2}$, then $\operatorname{WCS}\left(l_{M}\right)=\inf \left\{\inf \left\{c_{x, k}>0: R_{M}\left(k x / c_{x, k}\right)=\right.\right.$ $\left.(k-1) / 2\}: x=\sum_{i=1}^{n} x(i) e_{i} \in S\left(l_{M}\right)\right\}$.

Proof. Let $d=\inf \left\{\inf \left(c_{x, k}>0: R_{M}\left(k x / c_{x, k}\right)=(k-1) / 2\right\}: x=\sum_{i=1}^{n} x(i) e_{i} \in\right.$ $\left.S\left(l_{M}\right)\right\}$. 
For any $\varepsilon>0$, there is an $x=\sum_{i=1}^{n} x(i) e_{i} \in S\left(l_{M}\right)$, such that

$$
\inf \left\{c_{x, k}>0: R_{M}\left(k x / c_{x, k}\right)=(k-1) / 2: x \in \sum_{i=1}^{n} x(i) e_{i} \in S\left(l_{M}\right)\right\}<d+\varepsilon .
$$

So, there are $k>1$ and $c_{x, k}<d+\varepsilon$ such that $R_{M}\left(k x / c_{x, k}\right)=(k-1) / 2$. Put

$$
x_{m}=(\overbrace{0, \ldots, 0}^{m n}, u_{1}, u_{2}, \ldots, u_{n}, 0, \ldots) \quad(m=1,2, \ldots) .
$$

By the same method as in the proof of Theorem 3 , we have $x_{n} \stackrel{w}{\longrightarrow} 0$.

For any $k \neq n$, observe that

$$
\begin{aligned}
\left\|\left(x_{n}-x_{k}\right) /(d+\varepsilon)\right\|_{M} & \leq\left(1+R_{M}\left(k\left(x_{n}-x_{k}\right) /(d+\varepsilon)\right)\right) / k \\
& =\left(1+2 R_{M}(k x /(d+\varepsilon))\right) / k \\
& \leq\left(1+2 R_{M}\left(k x / c_{x, k}\right)\right) / k=1 .
\end{aligned}
$$

This means that $\left\|x_{n}-x_{k}\right\|_{M} \leq d+\varepsilon$, i.e., $A\left(\left\{x_{n}\right\}\right) \leq d+\varepsilon$. By the arbitrariness of $\varepsilon$, we have $\operatorname{WCS}\left(l_{M}\right) \leq d$.

Next, we will prove that $\operatorname{WCS}\left(l_{M}\right) \geq d$. Let $x_{n}=\sum_{i=I_{(n-1)}}^{I_{n}} x(i) e_{i} \in S\left(l_{M}\right)\left(I_{0}=\right.$ $0)$ be an arbitrary equidistant sequence such that $x_{n} \stackrel{w}{\longrightarrow} 0$. Take $k_{n, m}>0$ such that

$$
\left\|\left(x_{n}-x_{m}\right) / d\right\|_{M}=\left(1+R_{M}\left(k_{n, m}\left(x_{n}-x_{m}\right) / d\right)\right) / k_{n, m} .
$$

We will estimate $\left\|\left(x_{n}-x_{m}\right)\right\|_{M}$ by considering two cases:

I. $k_{n, m} \leq 1$. Then

$$
\left\|\left(x_{n}-x_{m}\right) / d\right\|_{M}>1 / k_{n, m} \geq 1,
$$

whence, $\left\|\left(x_{n}-x_{m}\right)\right\|_{M}>d$.

II. $1<k_{n, m}$. Without loss of generality, we assume that $n<m$. We have

$$
\begin{aligned}
\left\|\left(x_{n}-x_{m}\right) / d\right\|_{M} & =\left(1+R_{M}\left(k_{n, m}\left(x_{n}-x_{m}\right) / d\right)\right) \\
& =\left(1+R_{M}\left(k_{n, m} x_{n} / d\right)+R_{M}\left(k_{n, m} x_{m} / d\right)\right) / k_{n, m} \\
& \geq\left(1+\left(k_{n, m}-1\right) / 2+\left(k_{n, m}-1\right) / 2\right) / k_{n, m}=1 .
\end{aligned}
$$

Consequently, $A\left(\left\{x_{n}\right\}\right) \geq d$.

By the arbitrariness of $\left\{x_{n}\right\}$, it follows that $\operatorname{WCS}\left(l_{M}\right) \geq d$, which finishes the proof.

\section{REFERENCES}

1. W. L. Bynum, Normal structure coefficients for Banach spaces, Pacific J. Math. 86 (1980), 427-436. MR 81 m:46030

2. Guanglu Zhang, Weakly convergent sequence coefficient of product space, Proc. Amer. Math. Soc. 117 (1993), 637-643. MR 93d:46037

3. Toḿas Doḿinguez Benavides, Weak uniform normal structure in direction sum spaces, Studia Math. 103 (37) (1992), 283-290. MR 94c:46024

4. M. S. Brodskii and D. P. Milman, On the center of a convex set, Dokl. Akad. Nauk SSSR 59 (1948), 837-840. MR 9:448f

5. S. Chen, Geometric theory of Orlicz spaces, Dissertation Math., 1996.

6. R. Goebel and W. A. Kirk, Topics in metric fixed point theory, Cambridge University Press, 1990. MR 92c: 47070

7. F. Hiai, Representation of additive functional on vector-valued normed Köthe spaces, Kodai Math. J. 2 (1979), 300-313. MR 81d:46037 
8. T. C. Lin, On the normal structure coefficient and the bounded sequence coefficient, Proc. Amer. Math. Soc., 88 (1983), 262-264. MR 85g:46021

9. J. Lindenstrauss and L. Tzafriri, Classical Banach spaces, Springer-Verlag, Berlin, Heidelberg and New York, 1977. MR 58:17766

10. E. Maluta, Uniformly normal structure and related coefficients, Pacific J. Math., 111 (1984), 357-369. MR 85j:46023

11. J. Musielak, Orlicz spaces and modular spaces, Lecture Notes in Math., vol. 1034, SpringerVerlag, 1983. MR 85m:46028

12. M. M. Rao and Z. D. Ren, Theory of Orlicz spaces, Marcel Dekker Inc., New York, Basel and Hong Kong, 1991. MR 92e:46059

13. H. K. Xu, The Maluta problem of sequence constants in Banach spaces, Bull. Sci. China 34 (1989), 725-726. CMP 90:06

Department of Mathematics, Harbin University of Science and Technology, Harbin City, Heilonguiang 150080, People's Republic of China

E-mail address: cuiya@hkd.hrbust.edu.cn 